\title{
Démarche de co-conception citoyenne dans les processus d'innovation par les usages : le cas du projet Ecofamilies
}

\section{Approach to citizen co-design in the processes of innovation by uses: the case of the Ecofamilies project}

\author{
Franck Debos ${ }^{1}$, Céline Masoni Lacroix ${ }^{2}$, Natacha Cyrulnik ${ }^{3}$, Paul Rasse ${ }^{4}$, Brigitte Trousse $^{5}$ \\ ${ }^{1}$ Laboratoire SICLAB Méditerranée, Université Nice Sophia-Antipolis, debos.franck@wanadoo.fr \\ ${ }^{2}$ Laboratoire LIRCES, Université Nice Sophia-Antipolis, celilac@orange.fr \\ ${ }^{3}$ Laboratoire PRISM, Université Aix-Marseille, natacha.cyrulnik@wanadoo.fr \\ ${ }^{4}$ Laboratoire SICLAB Méditerranée, Université Nice Sophia-Antipolis, rasse@unice.fr \\ ${ }^{5}$ INRIA, Université Nice Sophia-Antipolis, Brigitte.Trousse@inria.fr
}

RÉSUMÉ. Les auteurs situent leur article dans un contexte d'innovation sociétale, qui place les usagers au centre de la démarche projet sous l'angle de la thématique énergétique. S'intéressant à une typologie des démarches de coconception existantes, ils proposent un retour d'expérience du projet participatif Ecofamilies, qui consistait pour des familles participantes, à co-concevoir et tester une solution technologique de suivi des consommations énergétiques domestiques, sur le plan des Interactions Homme-Machine, en adéquation avec leurs besoins. Les auteurs interrogeront les apports, les contraintes et les limites de l'engagement d'une démarche de co-conception selon la double perspective de l'évolution des usagers vers leur positionnement en tant qu'acteurs d'un projet collaboratif et les processus d'intelligence collective qui s'y déploient.

ABSTRACT. The authors place their article in a context of societal innovation, which puts users at the center of the project approach in terms of the energy theme. Interested in a typology of existing co-design approaches, they propose a feedback from the Ecofamilies participatory project, which involved participating families, co-design and test a technological solution for monitoring domestic energy consumption, The Human-Machine Interaction plan, in line with their needs. The authors will examine the contributions, constraints and limits of the commitment of a co-design approach from the perspective of the evolution of the users towards their positioning as actors of a collaborative project and the processes of Collective intelligence.

MOTS-CLÉS. Dispositif numérique, Co-conception, Eco responsabilité, Dépenses énergétiques, Usagers, Innovation, Démarche participative, Engagement.

KEYWORDS. Digital device, Co-design, Eco-responsibility, Energy expenditure, Users, Innovation, Participative approach, Commitment.

\section{Introduction}

Cet article a comme point de départ un double constat : l'intégration, voire l'intrusion sans cesse croissante, des technologies numériques dans les pratiques quotidiennes des individus, et la prise de conscience par les acteurs de l'innovation de la fertilité des réseaux de coopération informels, notamment la prise en considération des utilisateurs dans le processus de conception de dispositifs technologiques basés sur le numérique. À ce niveau, le concept d'innovation partagée (Duval, Speidel, 2014) semble se déplacer toujours plus vers l'usager.

L'objet de cet article est de montrer comment un dispositif de recherche centré sur un processus de négociation itératif entre des acteurs permet une plus grande adéquation d'une part d'un dispositif technologique numérique innovant et d'autre part de la vision managériale technophile de leurs concepteurs avec les attentes des usagers. Ces propos seront illustrés par le projet Écofamilies qui a consisté à prototyper et tester, à travers une démarche de co-conception, une solution technologique 
innovante contribuant à l'adoption de comportements écoresponsables chez des familles en termes de dépenses énergétiques. Ce projet s'inscrit dans le cadre du dispositif PACALABS sur une durée de quinze mois (novembre 2013 - mars 2015). Les partenaires étaient le CSTB (Centre Scientifique et Technique du Bâtiment) qui le coordonnait, le laboratoire I3M de l'Université de Nice Sophia Antipolis (SICLAB Méditerranée depuis 2017), la métropole Nice Côte d'Azur, les entreprises Ekenos (conception de tablettes) et Expérientia (design produit et d'interface) ainsi que le living lab «ICT Usage $\mathrm{Lab}^{1} »$ partenaire sponsor représenté par l'INRIA.

Cet article se décompose ci-dessous en trois parties. Tout d'abord nous traitons des liens entre les processus d'innovations et l'intégration des usagers au sein de la démarche d'innovation. Dans un second temps une section aborde la notion de co conception illustrée par le projet Ecofamilie. Pour finir une section éclaire l'importance des processus participatifs pour une meilleure connaissance des usages.

\section{Processus d'innovation et place de l'usager}

Avant de traiter de la démarche de co-conception, il semble important de présenter les grandes lignes de la démarche d'innovation en rapport avec l'usager. A la lumière de l'histoire simplifiée des formes d'innovation, nous pouvons voir que les individus ont, soit une vision « romantique » de l'innovation avec l'image du génie, du créateur solitaire et héroïque qui perdure depuis la renaissance soit une vision hyper rationnelle de l'innovation en tant que processus linéaire développé par de grands laboratoires et centres de R\&D où tout part de l'entreprise. Toutefois ces deux approches sont peu adaptées à une société centrée sur les services et la connaissance, se fondant de façon prépondérante sur les désirs et rêves des consommateurs (Guehika 2017).

En effet, et ce récemment, avec le fort développement du Web 2.0 voire 3.0, véritable espace public mondial de création sur Internet, l'innovation partagée entre le concepteur et l'utilisateur se déplace toujours plus vers l'utilisateur final. Internet a donc créé une transformation en profondeur des relations entre les individus et les organisations, alors que nous pouvons observer, depuis la fin des années quatre-vingt-dix, l'émergence successive du consom'expert, du consom'acteur et du consom'auteur (Maillet, 2008). Dans ce contexte, l'innovation partagée entre le concepteur et l'utilisateur se déplace toujours plus vers l'utilisateur final.

Il est important à ce niveau d'avoir une approche intégrative, voire holistique, de l'innovation (Biso, Le Naour, 2017) et de se rapprocher de l'idée de « co-innovention » développée par Pierre Musso, qui insiste sur l'aspect partagé entre des acteurs nombreux et diversifiés, ou encore de «l'innovention» selon Lucien Sfez, qui exprime le lien entre la science fondatrice qui invente, et la technique servante qui innove. De plus, les innovations n'ont pas toutes la même nature et le même degré d'importance, ce qui se traduit par plusieurs typologies intégrant, par exemple, principalement des dimensions commerciales ou encore l'impact sur les structures sociales d'accueil. Nous pouvons citer la classification d'Alter (2001) qui identifie :

- L'innovation ordinaire, qui correspond à la créativité des gens ordinaires dans la vie quotidienne (Akrich, 1998) les astuces déployées par les consommateurs dans le cadre de l'utilisation d'un produit ou d'un service. Se rattachent ici, également les travaux de Michel De Certeau qui insiste sur l'importance de la créativité des gens ordinaires. Pour ce dernier, les individus sont actifs et pas seulement soumis à la discipline de marché. Chaque personne va s'inventer une manière propre de consommer et celle-ci devient un lieu de créativité par le biais de bricolages avec les produits et les services ainsi que le recours à des ruses et des tactiques. Ces usagers agissent, de fait, de façon non prévue par les organisations marchandes.

\footnotetext{
${ }^{1}$ Le living Lab "ICT Usage Lab" de Sophia Antipolis a été le premier living lab français labélisé en 2006 par le réseau européen des livings labs (ENoLL). http://www.openlivinglabs.eu/livinglab/ll-ict-usage-lab 
- L'innovation incrémentale, conçue dans les services d'études et de recherches, qui est souvent une innovation de reproduction et qui demeure limitée.

- L'innovation de rupture ou stratégique qui engendre un nouvel ensemble socioculturel et économique.

Notre démarche, à partir du projet Ecofamilies peux se rattacher au processus d'innovation dit ordinaire et ascendant, car elle provient des utilisateurs eux-mêmes et non des entreprises, via leurs centres de Recherche et Développement (R\&D), à l'instar du logiciel libre qui est un processus d'organisation coopératif et bénévole.

\section{Encadrer un projet : de la co-conception à la collaboration}

\subsection{Principe de la co-conception et application à Écofamilies}

La co-conception est une démarche qui consiste à concevoir un produit ou un service avec l'aide et la participation active du client-consommateur-usager. Cela donne à ce dernier un rôle beaucoup plus important que celui de simple validateur dans le processus de conception du produit/service soit un rôle de co-créateur de valeurs (Foudriat 2016). Le résultat est ainsi non un produit/service parfait, mais un produit/service meilleur car plus pertinent et adapté aux attentes et contraintes des personnes participant à la démarche. L'atelier de co-conception permet la construction graduelle d'un consensus entre l'ensemble des acteurs concernés par ce projet. Cette méthodologie se distingue des approches plus traditionnelles dans lesquelles chaque étape est planifiée (analyse des besoins, conception de prototypes, essai en milieu réel, implémentation, évaluation, etc.) sans intégrer totalement ou partiellement les usagers et leurs pratiques. Elle se rattache à l'approche communicationnelle d'Habermas, à savoir un processus itératif préconisant le croisement des différents types de savoirs détenus par les acteurs impliqués. Dans ce contexte, il faut noter l'importance de l'« Actor Network Theory " (Esnault, Zeiliger, Vermeulin, 2006), permettant la prise en compte des influences potentielles d'acteurs de natures différentes, l'adoption d'un langage commun et l'acceptation de la nécessité d'aligner des intérêts pouvant être divergents selon les sous-groupes d'acteurs décris. Nous pouvons également retrouver cette démarche au sein de processus de communication engageante tel que décrit dans (Courbet $\&$ al, 2009).

Au niveau du projet Ecofamilies, l'usage se rapporte à l'utilité et à l'usabilité de l'outil : son utilité du point de vue des interactions de l'usager au système (des possibilités de contrôle et de remédiation que cet outil permet), son usabilité du point de vue de la facilité d'utilisation (et d'apprentissage) pour les utilisateurs. 


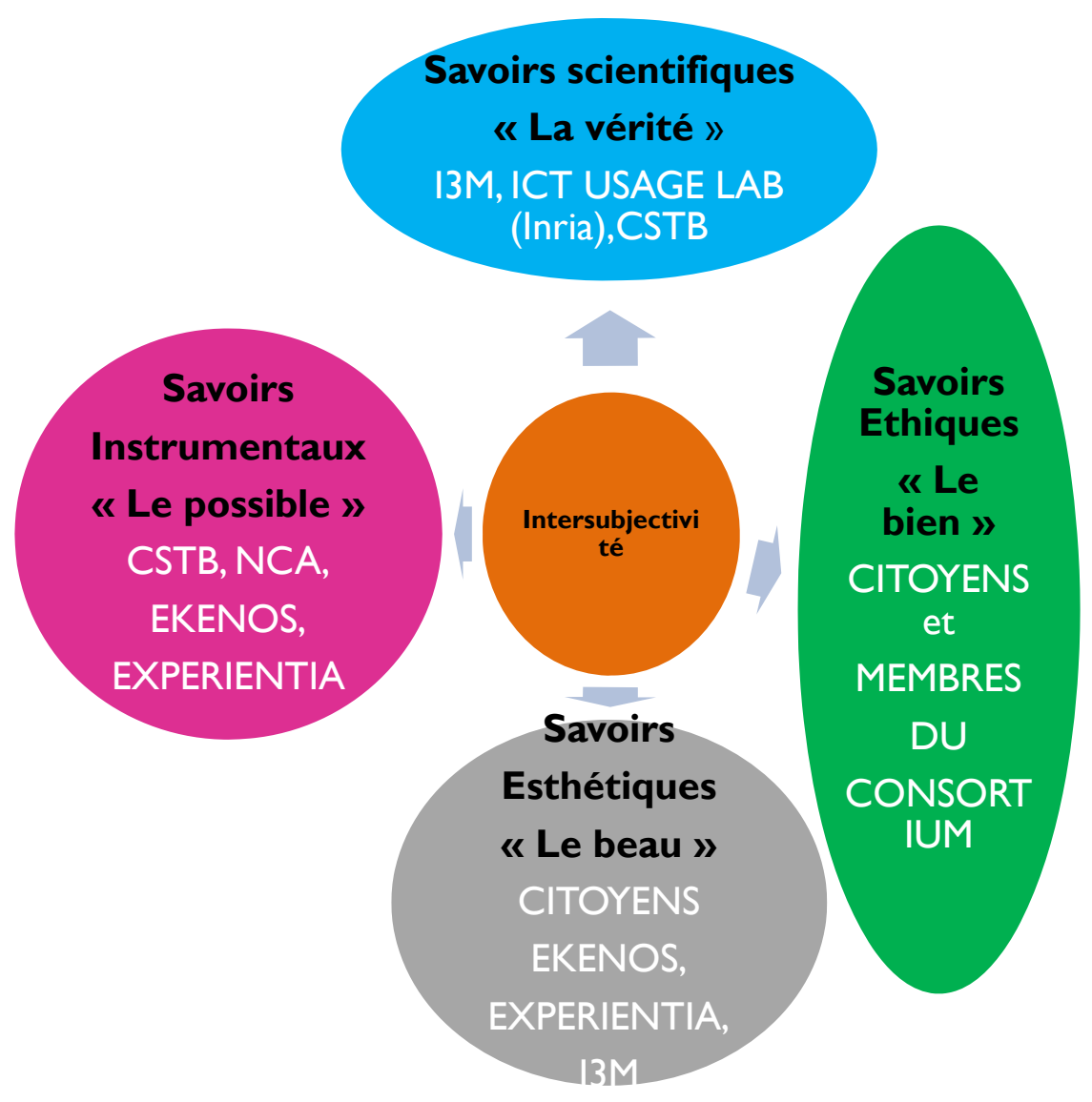

Figure 1. Schéma adaptant l'approche communicationnelle d'Habermas au projet Écofamilies

\subsection{La co-conception dans le cadre d'une démarche Living Lab}

Dans le cadre de notre démarche participative, il s'agit alors d'accompagner, par le biais d'ateliers collaboratifs de travail, les participants vers l'expression, voire le recadrage de leurs attentes vis-à-vis de la solution qu'ils ont à co-construire. Les participants sont ainsi informés des objectifs du projet, contextualisé par rapport à la question des économies d'énergie et des solutions existant sur le marché. Ils sont encouragés à donner leur avis sur les propositions de solutions alternatives émanant de l'entreprise de design d'interface, au moyen d'un réseau Internet de communication asynchrone (liste de diffusion, questionnaire en ligne, blog Ecofamilies) animé par un community manager, et lors de quatre réunions (ateliers de design/focus group) regroupant l'ensemble des partenaires, animées par les chercheurs, alternant les temps de travail dirigé avec des moments conviviaux d'échanges informels autour d'un cocktail buffet terminant chaque réunion.

Nous avons plus précisément construit, dans le cadre de cette démarche Living Lab, un dispositif socio-narratif, dans le sens où il engage des pratiques de narration d'usagers-participants, qui, à terme, génèrent une construction de sens par rapport à eux-mêmes, par rapport à la place que chacun occupe dans le projet, par rapport à la thématique générale du projet et à son implication sociétale. Ce dispositif se développe en trois étapes : des ateliers d'écriture de scénarios d'usage, un blog avec son forum et un film documentaire. Les familles participantes prennent ainsi part à des séquences itératives d'atelier de travail créatif, permettant aux chercheurs d'analyser de quelle façon elles interagissent avec un outil technologique, afin qu'il corresponde à leurs besoins réels. Les ateliers de scénario d'usage engagent les participants à raconter l'histoire de leurs habitudes de consommation « à la maison » sous forme d'intrigue, selon un schéma narratif quinaire, approprié dès les études secondaires et dont les cinq parties constitutives ont été expliquées et illustrées par les chercheurs-animateurs des ateliers. Deux situations leur sont proposées, l'hiver dans notre région méditerranéenne et un départ en vacances lors des congés de printemps. Ils doivent ainsi se représenter, pour chacun des deux 
contextes, quel serait l'outil, ou la solution technologique la plus adaptée à leurs besoins. La perspective narrative est renforcée par les appels à participation au forum, comme par les recueils de témoignages des participants, filmés à l'issue de chaque atelier, lors de séances informelles et conviviales.

L'approche narrative d'un projet participatif est confrontée aux limites de l'engagement des participants à écrire ou raconter des histoires sur différents supports médiatiques ; sur du papier lors des ateliers de scénarios d'usage, sur le Web avec le blog et le forum associé, ou face à une caméra avec le documentaire de fiction. La participation en ligne en est un problème récurrent, comme les « rôles » qu'endossent les participants dans les processus d'écriture ou filmique d'exposition de soi. Les outils socio-narratifs recueillent néanmoins les traces des interactions entre chercheurs et familles, qui se découvrent acteurs d'un même projet qu'ils co-construisent. Le film documentaire, dans lequel les participants décrivent leur engagement tout au long du projet, constitue un recueil de témoignages sur le processus de design participatif.

De manière générale, en racontant et en partageant leurs histoires, les familles mettent en perspective leurs habitudes de consommation et construisent une approche collective des besoins sociaux de maîtrise et de réduction des consommations énergétiques. A l'issue de la diffusion du film documentaire, lors du dernier atelier, les participants, chercheurs et familles, prennent de la distance par rapport à la situation dans laquelle ils étaient impliqués ; le processus de co-conception, créatif et social, peut être saisi dans son ensemble. 


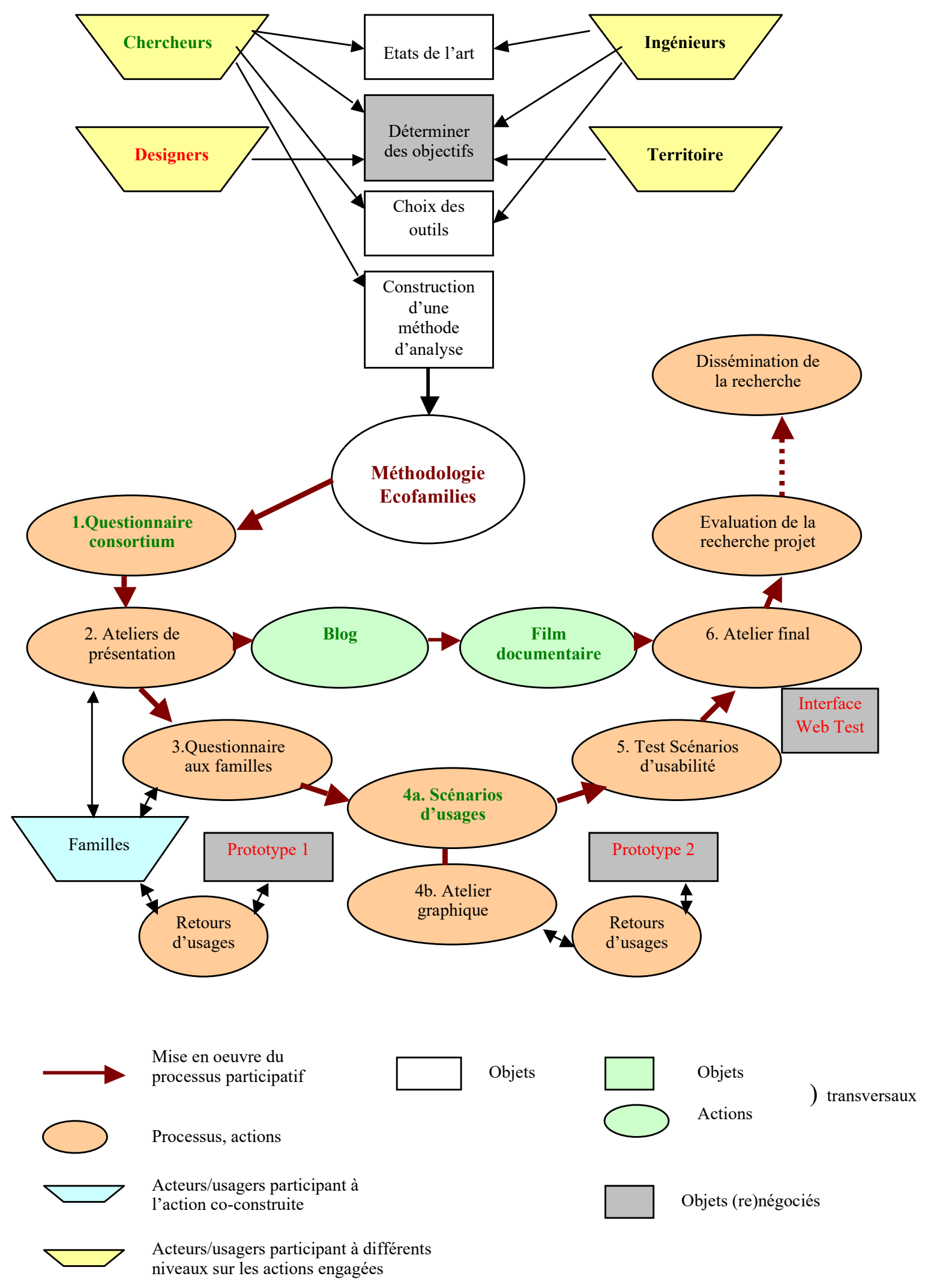

Figure 2. Cartographie de la méthodologie du projet Ecofamilies

Le graphique ci-dessus reprend les différentes étapes de notre dispositif participatif. En amont des premiers ateliers de présentation du projet aux participants, nous avons introduit un questionnaire interne au sein du consortium. Intitulé «Vers une culture commune», il interroge les différentes approches du projet, les connaissances préalables, les objectifs et les attentes des membres du consortium. Nous visons un accompagnement de la recherche, invitant les différents participants à partir d'une base épistémologique, si ce n'est partagée, pour le moins visant l'interdisciplinarité. Deux 
dimensions principales ont été déployées, qui ont permis de saisir les représentations scientifiques, sociales et culturelles de chacun en fonction de nos deux thématiques convergentes : la maîtrise de la consommation d'énergie et la recherche participative. Les différents participants partagent une construction similaire du projet ; il s'agit de définir la co-conception, de la nuancer, puis d'envisager son point central : les usagers et leurs usages, pour en venir à la thématique générale choisie : l'énergie, et enfin d' « évoquer » l'outil à co-construire. La question de la co-conception, prépondérante pour la définition générale du projet, s'efface au profit du design de l'outil numérique à co-concevoir, envisagé comme objectif pratique principal. Le questionnaire souhaite aussi interroger l'engagement d'une activité de recherche en lien avec les comportements citoyens des chercheurs. Il est à noter qu'en ouverture de projet, le rôle des membres du consortium, qui les définirait en tant qu'acteurs du projet n'est pas clairement posé. Alors qu'un niveau de consensus assez élevé est atteint, quant à l'implication éco-responsable (réelle ou fantasmée, pour le moins formulée) des différents membres. Ceux-ci s'en tiennent à l'expression de préférences individuelles et/ou de culture de groupe, dès lors que les questions concernent les implications sociales ou sociétales de ce type de projet. Trois tendances fortes sont ainsi à articuler : la démarche du projet engage une pratique de co-conception que les membres encadrant le projet ne se sont pas encore appropriés, la dimension pragmatique d'outil à co-construire prend une place prépondérante, enfin les acteurs du projet ne mettent pas nécessairement à distance leurs appréciations et jugements de valeur sur les implications sociétales que génère un projet participatif.

Notre méthodologie de projet instaure alors un cadre à la démarche participative de co-conception. Le premier atelier de présentation propose à nos participants un parcours découpé en trois sousateliers : présentation d'outils de mesure des consommations énergétiques existants, présentation et discussion autour des fonctionnalités attendues de la solution à co-construire, atelier participatif autour des éco-gestes. Dans la période de hiatus entre les deux premiers ateliers, notre dispositif encadrant la participation des familles volontaires installe un questionnaire en ligne et un blog afin d'entretenir la mobilisation et la motivation des participants. Le questionnaire, assez conséquent (130 questions), interroge les habitudes, les convictions et bonnes pratiques des participants, en matière de maîtrise de la consommation énergétique, liée à la question plus générale du développement durable, ainsi que leurs attentes par rapport à l'interface innovante à co-construire. Le blog, entretenu par un community manager, s'efforce de créer les conditions de l'interactivité. De simple support de communication, ou de lieu d'affichage du projet, il devient support d'animation, en proposant un espace collaboratif de type forum. Il persistera jusqu'aux derniers ateliers.

\subsection{Présentation du processus de recrutement ainsi que des quatre ateliers}

Dans le cadre du projet Écofamilies, le protocole suivant a été mis en place. À partir de la sélection et du recrutement d'une trentaine de familles, nous avons construit un dispositif expérimental autour de trois ateliers de co-conception itératifs, dont les résultats allaient permettre à notre partenaire designer Expérientia d'assurer différentes phases incrémentales de design d'une interface web, choisie par les familles dès le premier atelier.

\subsubsection{Processus de recrutement et profil des familles d'expérimentation}

Ce projet s'inscrit dans une démarche exploratoire qualitative et de fait, il est nécessaire d'avoir un profil bien diversifié sans soucis de représentativité (échantillon de jugement). Les sources de sélection des familles sont donc diversifiées et rattachées à l'ensemble des partenaires des acteurs du consortium concernés par cette phase de recrutement au sein du projet, notamment l'espace info énergie de NCA. Nous avons obtenu 46 réponses de particuliers intéressés par le projet et souhaitant y participer. Nous avons soumis les invitations de participation au premier atelier dès mi-février et cela jusqu'à début mars. Une vingtaine de familles ont été retenues, ce qui représente un total de 33 personnes. 
Le profil des familles recrutées est le suivant : la majorité (60\%) est propriétaire de leur logement et à ce niveau, l'ensemble des logements sont représentés, du studio à la maison (avec une prééminence relative des maisons). Les répondants ont quasiment tous entre 26 et 55 ans ( $40 \%$ entre 26 et 40 ans et $45 \%$ entre 41 et 55 ans. Ce sont des couples en grande majorité $(85 \%)$ ayant pour la plupart un à trois enfants $(60 \%)$. Nous pouvons aussi noter une surreprésentation des cadres et des professions intellectuelles (la moitié des familles). Les familles restantes se répartissent sur diverses catégories socio professionnelles (agriculteurs, artisans, employés, retraités, etc.). La valeur qui est commune à l'ensemble de ces familles est une réelle sensibilité aux problématiques de développement durable (voire un fort engagement pour les trois quart d'entre-elles) ce qui semble cohérent avec la thématique de cette recherche action.

\subsubsection{L'atelier 1 : ateliers de présentation}

Le premier atelier de présentation était un atelier plénier avec l'ensemble des familles qui proposait aux participants trois séquences de travail en sous-ateliers: sur les solutions technologiques existantes, sur les fonctionnalités envisagées de la solution technologique à co-construire, et sur les éco-gestes.

Le but de cet atelier était d'établir un premier contact avec les usagers participants à la coconception d'une interface de suivi énergétique. Cette première prise de contact a été l'occasion de présenter le projet, ses objectifs et d'éclaircir auprès des participants la notion de co-conception. Cet atelier a aussi permis de rassurer les familles sur les buts et les objectifs non lucratifs du projet, point d'inquiétude pour de nombreuses personnes présentes. L'atelier a été ensuite décomposé en trois sousateliers. Chaque sous-groupe (une dizaine de personnes) a pu participer tour à tour à un sous atelier nommé « Benchmarking Avantages/Inconvénients», un sous atelier nommé " Fonctionnalités \& services »ainsi qu'un sous atelier nommé « Eco-gestes ».

Les ingénieurs impliqués dans le projet avaient imaginé une tablette dédiée, spécifiquement conçue pour rassembler les données recueillies par les capteurs. Ils attendent que le collectif travaille sur le design, essentiellement la forme et l'affichage des tablettes, puis identifie le contenu des informations utiles aux usagers.

Mais, assez rapidement, le groupe "d'usagers" propose une solution radicalement différente : la conception d'une application regroupant ces informations pour les afficher sur les Smartphones et/ou sur les ordinateurs domestiques des habitants du lieu. Cela leur paraît beaucoup plus simple, plus pratique et plus économique, parce que mieux inséré dans leurs habitudes d'usage des technologies d'information. Cette solution a aussi le grand intérêt de permettre que les données puissent être consultées régulièrement en s'affichant automatiquement, ou à heures précises, ou pour signaler des pics de consommation, sur les écrans consultés régulièrement à la maison et à l'occasion de toutes sortes d'autres activités. En outre, les données peuvent être facilement conjuguées à d'autres informations en ligne, comme la météo, des conseils ou des écogestes, par exemple.

Il apparaît donc, à la lecture des réflexions et commentaires des familles d'expérimentation que les dispositifs et interfaces centrés sur le Web paraissent les plus adaptés pour mieux connaître, comprendre et agir sur la consommation d'énergie d'un foyer. Sur ce plan émergent globalement une orientation de besoins vers une application Web que l'on pourra trouver sur un site propre et/ou que l'on pourra intégrer sur un Smartphone, une tablette ou un ordinateur. Cette application sous-entend aussi un ensemble de capteurs dans le domicile permettant d'interagir sur la consommation d'énergie. L'outil dédié n'est pas apprécié.

Après le premier atelier et ce, dans la perspective du deuxième atelier, un questionnaire a été envoyé aux familles participantes pour nous aider à faire émerger chez elles des besoins et des attentes quant au nouvel outil. Le questionnaire qui a été ainsi soumis à ces familles, a été principalement élaboré 
pour fournir un maximum de matière exploitable et de données précises et suffisamment pertinentes aux designers chargés de la réalisation du prototype d'interface de sensibilisation énergétique.

Pour favoriser la synergie entre l'équipe projet, les concepteurs et les familles, il a été décidé de créer un espace communautaire sur le Web qui servirait de prolongement aux échanges initiés lors des ateliers, afin d'entretenir la mobilisation et la motivation des participants.

\subsubsection{L'atelier 2 : scénarios d'usage et approche graphique de la solution}

Le second atelier "Scénarios d'usage et approche graphique de la solution technologique » a été entièrement tourné vers la co-conception, avec l'élaboration de scénarii d'usage et de maquettes. Cet atelier a été organisé au cours de trois sessions différentes afin d'avoir des groupes de familles plus restreints, et ainsi permettre à l'équipe projet de consacrer plus de temps et d'établir une relation plus étroite avec chaque famille.

L'atelier croise une approche individuelle par l'écriture, à partir de scénarios d'usage proposés par les chercheurs, à l'expression graphique des fonctionnalités de l'interface. Les besoins et attentes, isolés lors de la phase narrative, sont restitués au groupe, en amont de l'atelier graphique, que les participants « imposent » d'ailleurs rapidement comme collectifs. Plusieurs scénarios sont construits autour des activités réelles, quotidiennes de l'usager en interaction avec l'interface, afin d'identifier les objectifs et besoins de l'usager, et plus finement la cohérence de ses besoins et de ce qu'il décrit de ses activités. Quel que soit l'atelier et le groupe et ce, malgré quelques disparités dans les avis et attentes, nous pouvons identifier des usages et des fonctionnalités du dispositif de maîtrise de la consommation énergétique, adaptés aux familles et à leurs membres et qui recueillent l'assentiment de l'ensemble des personnes interrogées.

D'après les participants, les informations essentielles à retrouver dans l'outil sont :

- La consommation électrique / eau / gaz (Globale et dans l'idéal par appareil).

- Les données météorologiques et la qualité de l'air (Données du milieu extérieur et dans l'idéal données sur la qualité de l'air intérieur).

- Des informations autour du déplacement et des transports (Données de trafic, outils de .covoiturage, émissions de $\mathrm{CO} 2$, horaires des transports en commun).

- Des conseils (éco gestes) adaptés à sa propre consommation (Afin de réduire ses consommations en adaptant son comportement, avec des contacts utiles ( $\mathrm{n}^{\circ}$ téléphone de réparateur, ...), ou liés aux alertes.

Les familles interrogées souhaitent également pour la plupart contrôler leur logement, ou tout du moins, avoir une information à distance sur les équipements (ouverture/fermeture des volets, régulation de la température, contrôle à distance des appareils électriques, contrôle des fuites d'eau et des anomalies de consommation, etc.). L'alerte semble aussi être un des besoins fondamentaux qui est ressorti lors de tous les ateliers. Pour ces derniers, l'alerte intervient pour mettre en avant un problème énergivore ou susceptible d'arriver. Cette alerte doit pouvoir être accessible notamment lors que l'occupant est à l'extérieur du logement (alerte météorologique, alerte de surcharge du réseau électrique, alerte en cas de consommation anormale d'appareils au domicile, etc.).

Pouvoir se situer par rapport à d'autres logements et d'autres familles est ressorti lors des ateliers du mois de mai. Cependant, ce point a fait dissensus au sein des participants qui n'ont pas tous accepté le principe de comparaison, jugé rédhibitoire par certains. Certains participants ont remis en cause la comparaison, trouvant difficile l'établissement d'une référence pertinente.

Pour finir, concernant la présentation des données, la majorité des participants souhaitent une interface simple, avec des icônes repérables facilement qui regroupent le même type de données, sur 
lesquelles on peut cliquer pour accéder à plus de détails. Les couleurs doivent faire ressortir les données importantes (indicateur vert quand tout est normal, rouge en cas d'anomalie) avec des icônes repérables facilement qui regroupent le même type de données, sur lesquelles on peut cliquer pour accéder à plus de détails. Les données doivent être hiérarchisées (notion d'arborescence) : du global (affiché en première page) au détail (quand on clique sur la donnée globale, si on souhaite aller plus loin) par catégorie d'appareils, par pièces, par capteurs. L'interface doit être interactive et pédagogique et adaptable aux différents supports (écran d'ordinateur, tablette, téléphone portable).

Un besoin fort des familles d'expérimentation a été la volonté d'avoir un outil qui s'adapte au logement de chacun et donc par conséquent facilement programmable, paramétrable et personnalisable par l'utilisateur. Les besoins qui sont ressortis sont :

- La possibilité de créer et personnaliser son interface en le composant à partir de widgets, en fonction de ses besoins, de son mode de vie, du contexte saisonnier et climatique, de ses moyens et le cas échéant des capteurs installés dans l'habitat.

- La possibilité de programmer des scenarii permettant d'anticiper des situations inhabituelles et de programmer les équipements en conséquence (départ en vacances, vague de froid, ...).

A l'issue de ce travail, le premier prototype réalisé par l'équipe des designers en fonction des retours d'usage (premier atelier et questionnaire) est proposé à l'attention aiguisée des usagers-participants. Deuxième hiatus, toujours animé par le blog dédié au projet et troisième atelier, où le second prototype, enrichi des retours d'usages des ateliers de design (scénarios d'usage et graphique) et du blog est présenté aux participants dans le cadre de tests d'usabilité conçus par l'équipe de designers.

\subsubsection{L'atelier 3 : test scénarios d'usabilité}

Avec les résultats de l'atelier 2, Experientia a élaboré un prototype d'interface web correspondant mieux aux besoins des participants qui ont émergé lors des deux premiers ateliers. Le troisième atelier a été l'occasion d'un débat autour de ce travail pour voir les améliorations à donner au produit et les pistes vers lesquelles faire évoluer la solution dans le futur. La soirée a été structurée autour du test du prototype par les familles suivi d'une séance libre de discussion avec Experientia. Dans ce contexte, Experientia a délivré un véritable prototype d'interface, sous la forme d'un ensemble de pages Web qui intègre les idées et commentaires principaux émis par les familles lors de ce dernier. Cet atelier a été l'occasion d'un débat pour observer et tenter de décrypter les réactions des familles face à la solution technologique présentée ainsi que de faire découvrir aux familles un dispositif de captation des comportements visuels sur un écran, de tester une partie de l'interface proposée par Experentia avec certains d'entre-deux et de restituer les résultats d'analyse issus du logiciel d'eye tracking (plateforme FocusLab du ICT Usage Lab) qui ont permis de retrouver/préciser certains problèmes évoqués par les participants.

\subsubsection{Le film documentaire présenté lors de l'atelier final}

Tout au long du projet, une documentariste a filmé le processus et les participants ont été conviés à une projection-débat du film documentaire du projet ainsi qu'à la visite du laboratoire expérimental Gerhome du CSTB, où le logiciel test co-conçu avait été installé pour simuler un contexte réel.

Réalisé par Natacha Cyrulnik (Chercheur au laboratoire I3M lors du déroulement du projet), le film documentaire (39'40) du projet Ecofamilie permet de retracer l'implication des familles dans le projet à travers leur participation aux trois ateliers de design participatif. Ce film a été présenté aux familles mais aussi à un certain nombre de représentants d'organismes de la Région invités pour l'occasion lors de l'atelier de clôture du projet organisé au CSTB à Sophia Antipolis. Il a donné lieu à un débat, dont la synthèse est proposée ci-après. 
Deux dimensions ont ainsi été soulignées par les participants ; le film documentaire de Natacha Cyrulnik a su retracer un processus, illustrer une démarche et respecter le naturel des participants ainsi que leur esprit critique. Les participants se sont aussi exprimés sur l'intérêt de la démarche de coconception, abordant principalement la définition de ce projet collectif et l'impression générale que l'accompagnement des chercheurs avait pu leur laisser.

La dimension collective et participative du projet a été affirmée et accompagnée d'un sentiment de plaisir à travailler ensemble. Les participants ont aussi dépassé cette dimension plus affective de la participation, vers une vision plus conceptuelle, puisqu'il s'agissait de : «Travailler ensemble » (répété par plusieurs participants), mais encore de " processus d'intelligence collective », concept ayant reçu l'assentiment de la majorité du public qui a estimé comme extrêmement stimulant de travailler ensemble sur un projet de création. Création, le mot est lâché car ce qui explique aussi l'implication des familles d'expérimentation à participer à ces ateliers consiste en la possibilité de concevoir ensemble un nouveau service, voire un nouveau dispositif en adéquation avec leurs attentes (Simon, Cohendet, Sarazin, 2017). Enfin, les participants ont été sensibles à la qualité d'écoute de l'équipe de chercheurs, ils ont considéré que leur parole était prise en compte. Dans le même ordre d'idée, ces remarques spontanées à l'issue du projet, illustrent bien l'importance de l'empathie avec le public qui peut s'opposer à un comportement de distanciation avec le public que l'on peut retrouver dans ce type de démarche de recherche lorsque le chercheur veut faire valoir son expertise par une certaine hauteur de comportement (celui qui sait) et ce, même inconsciemment. En effet, cela va bien au-delà de la seule qualité d'écoute, car d'après plusieurs participants, c'est également le comportement des membres du projet entre eux qui reflétait une complicité et proximité relationnelle forte, c'est le langage utilisé par l'équipe de recherche lors des ateliers qui était très proche des familles d'expérimentation qui a permis cette " création d'ambiance » (que l'on peut assimiler à des actions de communication sensorielle) et entraîner une dynamique de groupe réelle et forte, facilitatrice de la démarche de co-conception.

Cela peut laisser entendre que dans le cadre de ce type de dispositif sociotechnique de co-création, le premier niveau de création est cet univers de convivialité, de réelle connivence indispensable pour les familles qui l'ont exprimée à toute démarche de co-conception réussie. En d'autres termes, d'un point de vue extérieur, la démarche de co-conception se teinte d'optimisme « idéologique » (« très joli projet, mais... ») et l'intervention, voire l'intrusion directive de l'équipe de chercheurs, c'est-à-dire encore leur arbitrage a été interrogé et visiblement perçu comme inévitable. Alors que les participants à la démarche ont su équilibrer leur relation « affective » au groupe, ce sentiment positif du travailler ensemble, en s'accordant pour le dépasser vers une évaluation plus conceptuelle de la co-conception.

Il faut néanmoins rajouter le fait que bien que les familles qui ont participé au projet aient eu un ressenti globalement positif, elles ont également noté qu'il existait toujours un décalage entre leurs attentes et la production finale des designers d'Experientia, ce qui illustre bien le décalage, très difficilement évitable entre la culture technophile des designers avec les attentes réelles des participants. Ce processus pourrait aussi être envisagé sur une durée plus longue avant d'arriver à un consensus à ce niveau. Cela met donc de nouveau en exergue l'importance de la création de liens forts et vrais avec les familles d'expérimentation si cette démarche de co-conception doit être envisagée sur une période plus longue.

\subsubsection{Recommandations globales.}

À partir de ces réflexions, nous pouvons donner trois recommandations qui semblent importantes pour la réussite de ce type de projet.

- Dans le cadre de ce type de dispositif sociotechnique de co-création, le premier niveau de création est cet univers de convivialité, de réelle connivence indispensable entre l'équipe du projet et les familles participantes, condition qui semble nécessaire pour toute démarche de co-conception réussie. 
- La dissonance persistante entre la production des designers et les souhaits des familles impliquées dans le processus, met l'accent sur le fait que cette démarche doit être envisagée sur une durée plus longue avant d'arriver à un consensus à ce niveau. Cela met donc de nouveau en exergue l'importance de la création de liens forts et vrais avec les familles d'expérimentation si la démarche de co-conception doit être envisagée sur une période plus longue.

- Il apparaît également l'obligation de développer une culture commune en regard de cette démarche de co-conception au niveau des acteurs en présence, afin de respecter jusqu'au bout la dimension participative et de prendre conscience que ce type de démarche doit être envisagé sur du moyen terme.

\section{Usages, usagers et processus participatif}

\subsection{Analyse du processus participatif d'Ecofamilies}

Dans le cadre de ce projet pluridisciplinaire réunissant ingénieurs, designers, chercheurs et agents d'une collectivité territoriale, une démarche participative de co-conception d'un outil technologique de suivi énergétique adapté aux besoins des participants a été mise en œuvre.

Les contraintes temporelles nous sont apparues prégnantes dans le déroulement de nos ateliers. Lorsque le rythme de travail dans certains ateliers était trop élevé, lié à des contraintes de résultats rapides, il empêchait l'instauration de la démarche participative et entraînait plutôt le mécontentement des participants. En revanche, les ateliers de scénarios d'usage et graphique, découpés en sous-groupes, laissaient le temps de la mise en œuvre aux participants et produisait un sentiment d'appartenance à un groupe et d'implication dans un projet. Du côté de l'équipe d'animation, ces ateliers de co-conception scripturale et graphique étaient, de même, considérés comme construisant la démarche participative visée.

Il nous est ainsi apparu intéressant d'analyser une situation empirique au sein d'un groupe social restreint afin de mettre en question de manière concrète le processus de négociation. En d'autres termes, nous nous sommes intéressés aux manières dont les individus appréhendent une situation empirique posée, comment ils se forgent une opinion en fonction de leurs habitudes, de leurs pratiques et de leurs connaissances et comment ils diffusent ces connaissances et pratiques vers d'autres individus, qui proposent leur propre mode d'appréhension de la situation. Nous nous sommes alors demandé ce que ce phénomène de communication, accompagné de la médiation ou de l'animation de notre équipe de chercheurs génère, du point de vue de la formation d'un groupe et des échanges démocratiques qui peuvent s'y produire, du point de vue aussi de la transformation de ces habitudes individuelles en pratiques collectives, phénomène itératif, que nous qualifions volontiers de processus de négociation et renégociation.

La co-construction de notre dispositif participatif articulant ateliers de travail, moments d'échanges informels, processus filmique et débats, nous a permis d'associer nos familles participantes à un processus, sur lequel ils avaient en outre la possibilité de prendre de la distance critique et de la formuler, de la partager avec le groupe.

La dimension participative du projet s'affirme centrale ; elle a occupé avec les ateliers de scénarios d'usage et graphique et la projection-débat du film documentaire le cœur du projet. On pourrait considérer que la transition d'ateliers semi-directifs vers des ateliers participatifs assure de la cohérence à la démarche engagée. Le troisième atelier, directif, pourrait être perçu comme un retour en arrière, à des pratiques non-participatives de l'échange, voire à une négation ou à une rupture du processus d'engagement des publics. L'atelier ultime a vu coexister des perspectives directives et participatives... 
Une démarche participative ne consiste pas en une succession, voire une alternance de pratiques directives ou semi-directives et participatives. Plus finement, nous pouvons avancer qu'en fonction des attentes et des contraintes des différentes entités qui se sont « associées » pour ce projet, des chercheurs en sciences de l'information et de la communication ou en usages des technologies, des ingénieurs et des designers, le projet de recherche a été appréhendé à des niveaux différents, qui pouvaient coexister, voire se rencontrer et bénéficier de cette rencontre, ou œuvrer de manière parallèle, voire antagonique. En d'autres termes, la démarche projet a elle-même subi les rivalités antagoniques de tout processus démocratique délibératif, sur lequel nous revenons ci-dessous, où les prises de positions de « minorités » culturellement différentes peuvent être entendues et participer de ce processus.

Il faut encore préciser que le dispositif de co-conception a été installé en toute autonomie par les membres du consortium, il ne ressortissait ni d'une commande institutionnelle, qui aurait pu gérer sa mise en œuvre, ni d'une initiative citoyenne qui aurait pu désirer faire entendre sa voix sur les problématiques énergétique ou sociétale. Le dispositif de co-conception est issu d'une proposition mise en forme de manière interdisciplinaire et évolutive par une équipe de recherche (rapidement mue en équipe d'animation), et qui en a appelé à la participation de citoyens.

L'étude du dispositif de co-conception, descriptive et critique visait alors l'étude des usages et des usagers du projet. En d'autres termes l'étude du dispositif, de sa mise en œuvre et de son suivi, n'a de sens qu'en tant que ce dispositif est destiné aux pratiques et aux usages de publics engagés dans un projet. Ces différents éléments convergent vers la question du pouvoir des sciences sociales et de leurs méthodes. John Law et John Urry (2003) avancent que les changements physiques et sociaux dans le monde sont parallèles (et doivent l'être) de changements dans les méthodes de l'enquête sociologique. Les sciences sociales ont toujours besoin de se recréer, de renouveler leurs méthodes. Leur argument est que les méthodes de recherche sont performatives ; elles ont des effets, elles transforment les événements et produisent aussi, en un sens, ce qu'elles découvrent.

$\mathrm{Du}$ point de vue du chantier Ecofamilies, nos ateliers de co-conception participent d'un renouvellement méthodologique des sciences sociales, qui va avoir un effet sur les usages de la solution technologique à co-concevoir et sur l'engagement des usagers dans ce travail de coconception. Une dynamique scientifique et sociale se fait jour. Elle interroge nos pratiques scientifiques, citoyennes et démocratiques. En effet, lors des différents ateliers, une évolution, voire une transformation notoire de l'approche de notre objet de la part des participants s'est dévoilée.

\subsection{L'expérience collaborative au sein d'un projet en mode Living Lab}

Dans cette expérience de co-construction, les points de vue exprimés par les participants varient en fonction des conditions d'existence (les revenus plus ou moins confortables de la famille, le fait de vivre seul ou de voyager pour des raisons professionnelles et donc de laisser son habitation libre plusieurs semaines d'affilées), le type d'habitation (maison individuelle ou appartement), des modes de vie, ou en fonction des clivages de genre, d'âge, ou de statut social (celui de femme/homme, de manager/employé, de technicien ou ingénieur/retraité ou femme au foyer, ou encore d'étudiant, etc.). Mais les clivages de statut socioprofessionnel, de sexe, de génération sont rapidement dépassés vers la prise en considération de points de vue différents, en fonction des histoires, des préoccupations, des aspirations des uns et des autres. Ce dispositif collaboratif permet aussi de mobiliser des expériences de vie, sociales et professionnelles, des savoirs, des savoir-faire, des talents différents.

Si ces différents éléments, caractérisant les différents profils des participants, ont des implications sur les demandes et les suggestions qui sont faites, nous avançons que cette diversité peut être mobilisée en vue d'une expérience collaborative. Plus précisément, nous avons pu isoler trois profils croisés de participants, en fonction de leur positionnement en termes de coût, de valeur ou de compétences technologiques : 
- L'imaginatif versus le pragmatique : les aspirations et demandes articulent ici les potentialités de la solution technologique et ses traductions en termes de coût et de faisabilité.

- Le militant versus le prévoyant, qui accordent la prégnance aux aspects éthiques et solidaires ou aux alertes et à la possibilité d'agir à distance.

- Enfin le technophile versus le " technophobe », qui est intéressé par toute innovation technologique, si sophistiquée et complexe soit elle, ou privilégie au contraire des commandes, un affichage, une ergonomie visant la simplicité et correspondant à l'adaptation de leurs usages.

Dans un processus collaboratif, ces différences, ces dichotomies semblent s'effacer au profit d'un phénomène d'écoute et de partage, condition sous-jacente à la co-construction d'une solution pouvant satisfaire les besoins et les attentes du plus grand nombre.

Le phénomène prégnant dans le déroulement des ateliers gagne, selon nous, à être saisi par une ethnographie narrative et communicationnelle ; chacun se montre attentif aux remarques et suggestions d'autrui. La plupart s'efforcent même d'obtenir l'assentiment du groupe. Tous explicitent leurs points de vue et confrontent des solutions, creusent les pistes esquissées par d'autres, puis négocient pour trouver des compromis. A l'issue de la discussion, les points de vue convergent, intègrent les attentes des autres participants, se font plus complexes, et en même temps plus pragmatiques, en prenant notamment en considération les coûts et les conditions de réalisation. Les suggestions jugées superficielles (gadgets), sans véritable intérêt ou irréalisables (pour des raisons techniques ou de coût) sont abandonnées. Les solutions proposées deviennent plus cohérentes. On passe d'un vaste panel d'attentes confuses, diversifiées mais peu structurées, peu motivées et peu engagées, à une série de demandes jugées les plus essentielles et réalisables, mieux argumentées, plus convaincantes.

On a ainsi vu émerger face aux attentes des ingénieurs, enclins à imaginer des solutions ingénieuses mais d'utilisation complexe, sophistiquées mais onéreuses (pour le moins avant leur généralisation et leur production en masse), celles d'usagers solidaires, conscients des coûts, du gaspillage, des dimensions superficielles de certaines propositions, soucieux de produire des technologies accessibles à tous, de prendre en considération les moyens financiers, les niveaux de compétence et de mobilisation des différentes catégories d'usagers.

Il ne s'agit donc plus de mettre en œuvre un projet conçu en amont par une équipe restreinte de spécialistes, mais de le développer en créant des synergies de travail entre des chercheurs de statuts très différents et des citoyens usagers-participants, au moyen d'un dispositif articulant un réseau Internet de communication asynchrone et un système d'ateliers de design synchrone. Le dispositif de coconception s'affirme en tant que pragmatique de l'innovation. Il permet, d'une part, d'envisager la connaissance de l'environnement cognitif, technologique, social dans lequel doit s'insérer le projet d'innovation, non pas seulement d'un point de vue théorique et extérieur, mais de façon introspective, au fur et à mesure que les solutions technologiques sont abordées, discutées, repoussées ou retenues. Les solutions se construisent progressivement à partir des suggestions et des discussions qui s'ensuivent, quant à leur faisabilité, à leur coût, mais aussi en fonction des récits et de l'argumentation des acteurs, qui pour gagner l'assentiment des co-concepteurs se projettent, envisagent leur utilisation dans la vie quotidienne diversifiée de chacun.

\section{Conclusion: Vers une reconfiguration des pratiques des chercheurs : une pragmatique de l'innovation?}

Lors des ateliers de co-conception, en écriture et design, les chercheurs ont vu leurs rôles évoluer vers des pratiques de médiation et d'accompagnement, qui sous-tendent voire justifient le cadre qu'ils « imposent » aux participants du projet. Plus précisément, dans les ateliers de scénarios d'usage et de design, les chercheurs sont partagés en animateur/modérateur et en observateurs. Le principe d'animation des ateliers, variante de la pratique des Focus Groups, suppose que les intervenants aient 
un rôle qui aille du moins au plus directif. Autrement dit, les règles de fonctionnement peuvent être relativement floues au début du travail en groupe, de façon à faciliter les interactions, les interprétations divergentes, tout en étant révélatrices de la conception que les participants se font du sujet, puis être progressivement précisées.

De manière plus générale, poser un cadre à notre projet participatif, mais accepter qu'il puisse être co-construit, renégocié par les participants, nous permet d'affiner notre méthodologie de travail, mais encore et de manière plus durable, d'envisager certaines mutations de nos méthodes et pratiques de chercheurs à l'instar de pratiques collaboratives et artistiques associées à des processus qualitatifs ou quantitatifs plus académiques.

La fertilisation de nos méthodes et pratiques de recherche en une approche communicationnelle, est essentiellement fondée sur les relations et les interactions entre individus et générant un collectif. Les approches ethnographiques, par la narration, le design, le film, la pratique d'ateliers ou la participation à des forums en réseau avancent que la culture, le savoir, comme les savoir-faire sont des compétences à acquérir et à développer. La construction ethnographique doit être entendue comme une approche qualitative d'usages et de compétences, situés et contextualisés.

Il s'agit de requalifier la relation des usagers à la technique, aux technologies et aux objets, produits ou services à co-construire. Les retours d'usage des familles engagées dans le projet ont rapidement décidé de l'abandon de l'objet technologique, type tablette, au profit d'un service dédié. Nous avançons que les citoyens en situation collaborative de conception déploient une compétence " d'économie technologique ». Les usagers engagent ainsi une technologie dans le social, interrogeant son efficacité pratique en lien avec des usages réels, « simplifiés » par la technique.

Il s'agit encore de requalifier une certaine idéologie de la construction collective de connaissance, en évaluant la production de savoirs, l'acquisition de savoir-faire et la mobilisation de compétences. Il faut aussi s'interroger sur leur caractère formalisé ou stabilisé, formel ou informel et sur la qualité de ces savoirs ou compétences engagés dans un processus de co-constrution. Les usagers citoyens participant au projet Ecofamilies, citoyens lambda, n'accueillent parmi eux ni Lead-Users (Von Hippel, 1986) ou expert, ni développeur-amateur ; ils ne possèdent pas les compétences techniques des designers ou des ingénieurs. Nous pouvons nous interroger, avec Josiane Jouët (2000) sur l'apport des usagers dans la co-construction d'une solution technologique adaptée à leurs usages, et qu'il nous a fallu déterminer en amont, afin de dépasser la dichotomie entre besoins et usages fantasmés et besoins et usages réels. Le design participatif, la co-conception se découvrent ainsi non essentiellement attachés aux savoirs et savoir-faire technologiques, mais plus intimement aux compétences informelles et collaboratives que peuvent développer des individus en situation de co-création. Ce qui signifie aussi, paradoxalement, que leur créativité a dû et doit être encadrée, accompagnée dans un dispositif de recherche contextualisé, adapté à la situation de co-conception envisagée.

Dans les ateliers, animateurs comme observateurs ont pu saisir l'utilisation de ces compétences à différents niveaux d'appropriation, voire leur absence, tout autant que la possibilité de combler certaines « carences » dans l'élaboration et la négociation collectives de préconisations ou de solutions. Plus précisément, la compétence narrative que nous avons pu voir se développer au sein des ateliers de scénarios d'usage engage, sur la base d'une structure narrative acquise, des récits individuels d'expérience visant le partage, puis la mise en commun d'usages appropriés, distanciés par la narration. Ce processus narratif mobilise des interactions nourrissant une vision partagée. On ne peut affirmer qu'une identité collective productrice de sens, et qui pourrait être définie comme une communauté épistémique et/ou de pratiques émerge. Mais on ne peut nier un engagement du collectif. Des compétences relationnelles, se déploient, des qualités d'écoute et d'intégration des préoccupations individuelles, qui s'articulent dans un " agencement » collectif. En d'autres termes, au sein de ce dispositif cognitif et social, des compétences individuelles croisées prennent forme, en amont d'un 
processus de formalisation. L'accompagnement narratif des co-concepteurs joue, en effet, sur des compétences acquises et à développer ; elles pourraient conduire à l'expression et l'utilisation de compétences discursives, en germe dans la mise en discussion des récits d'expérience de chacun. L'argumentation, la mise en avant d'arguments et de contre-arguments s'entendent comme les compétences discursives "légitimes », dans le cadre de l'échange démocratique, ainsi qu'a pu le développer Habermas (1988), voire dans la prise de décisions politiques et territoriales, qui représentent bien le niveau « hiérarchique » supérieur d'un processus engageant des citoyens impliqués dans des problématiques environnementales.

Cette reconfiguration de la recherche orientée usages et usagers dévoile un processus de coconstruction de connaissance et de sens à partir de la mobilisation et du partage de compétences. Elle n'est pas sans effet sur la place du chercheur au sein du dispositif. Il s'agit de pénétrer un phénomène et de l'envisager comme processus à la fois instable et réflexif. Le chercheur modèle son objet de recherche et se trouve remodelé par lui. Le chercheur met en œuvre une méthodologie de recherche dans un environnement incertain ; une méthodologie qu'il aura à négocier, envers lui-même, envers ses pairs et envers les co-concepteurs, de manière itérative, tout au long du processus de recherche. L'interdisciplinarité peut être le gage d'une saisie d'un environnement de recherche complexe et mouvant. Elle autorise l'appréhension d'interrelations complexes, au sein d'une approche communicationnelle réflexive, qui déploie des perspectives ethnographiques qualitatives et compréhensives convergentes. Ces ethnographies croisées, en actualisant des approches narratives, graphiques, filmiques, présentielles et médiées, offrent un cadre à notre étude d'usages et concourent à la saisie, si ce n'est à la maîtrise, de la génération d'un collectif, où les individus s'inscrivent dans un processus de participation puis de collaboration.

On peut donc considérer qu'une communauté de pratiques s'est développée, sur le modèle de Wenger (Soulier, Audran, 2017) et que cette communauté est fédérée par un projet commun, avec des processus collaboratifs basés sur la participation et un système d'intérêts cohérents, vers la coconstruction d'une culture d'échange, voire de réciprocité. Nous retrouvons également les facteurs clés de succès isolés par Wenger : des outils simples, une convergence entre l'intérêt individuel et l'intérêt collectif, des résultats utilisables à court terme, ce qui se traduit dans nos ateliers par une traduction rapide en avancées technologique et de design, enfin de l'animation. Dans le cadre de nos ateliers de scénarios d'usage, les participants ont pu reformuler leurs besoins réels en matière de maîtrise de leurs consommations énergétiques, en racontant comment ils pouvaient faire face à telle ou telle situation de dépense et d'économie d'énergie scénarisée. Exposer son histoire personnelle et entendre l'histoire d'autrui est déjà co-construire un récit commun, qui permet d'envisager des solutions partagées, bénéfiques au collectif en cours de formation. La narration, puis la diffusion de leurs propres scénarios engagent les individus non seulement à former un groupe, voire une communauté, mais encore à négocier et renégocier, en fonction de leurs propres attentes et des connaissances que ce collectif naissant génère, les fonctionnalités techniques et d'usage de l'objet ou de la solution technologique qu'ils ont à co-concevoir.

Notre positionnement en sciences de l'information et de la communication nous a permis d'interroger de manière critique la question des usages : de nos représentations et pratiques de la recherche à notre positionnement en tant qu'animateur d'un groupe de participants citoyens, vers la démarche de co-conception à laquelle les sujets expérimentaux ont participé, qu'ils ont évalué et qui a modifié leur appréciation d'un chantier de recherche expérimental, leur appréciation de la dimension collective du travailler ensemble, qui a enfin mis au jour leur capacité délibérative de reformulation et d'adaptation de leurs besoins énergétiques individuels et, en fonction du collectif, leur capacité à la négociation. 


\section{Bibliographie}

[AKR 98] AKRICH M., «Les utilisateurs, acteurs de l'innovation permanente », Éducation permanente, n¹34, Paris, p. 79-89, 1998.

[ALT 01] ALTER N., L’innovation ordinaire, PUF, Paris, collection Quadrige, 2001, 284 pages.

[BIS 17] BISO S, LE NAOUR M., Design Thinking - Accélérez vos projets par l'innovation collaborative, Dunod, 160 pages, 2017.

[COU 09] COURBET D., BERNARD F., JOULE R V., HALIMI-FOLKOWICZ S., " Communication numérique engageante et protection de l'environnement : effets immédiats et différés sur les comportements, l'attitude et la mémorisation », 3ème Colloque International de Psychologie Sociale et Communication, p. 142-144, 2009.

[DEC 04] DE CERTEAU M., L'invention du quotidien, Tome I, Arts de faire, Gallimard, Folio Essais, Paris, 287 pages, 2004.

[DUV 14] DUVAL M., SPEIDEL K., Open Innovation, Dunod, Paris, 186 pages, 2014.

[ESN 06] ESNAULT L ., ZEILIGER R ., VERMEULIN F., "On the Use of Actor-Network Theory for Developing Web Services dedicated to Communities of Practice", TEL-CoPs'06 Proceedings, Palette, Crete, Greece, p. 80-88, 2006.

[FOU 16] FOUDRIAT M., La co-construction : une alternative managériale, Presses de l'EHESP, Paris, 193 pages , 2016.

[GUE 17] GUEHIKA A., Cartographie des processus d'innovation, Amazon Média, EU, 137 pages, 2017.

[HAB 84] HABERMASS J., The Theory of and Rationalization of Society, Beacon Press, Boston, 465 pages, 1984.

[HAB 85] HABERMAS J., The Theory of Communication Action, In Lifewordans System: A Critic of Functionalist Reason, Boston, 457 pages, 1985.

[HAB 88] HABERMAS J., L'espace public : Archéologie de la publicité comme dimension constitutive de la société bourgeoise, Payot, Collection : critique de la politique, Paris, 322 pages, 1988.

[JOU 00] JOUET J., Retour critique sur la sociologie des usages, Réseaux, vol. 18, nº 100, p. 487-521, 2000.

[LAT 91] LATOUR B., CHARVOLIN F., SCHWARTZ C., « Crise des environnements, défis aux sciences humaines », Futur Antérieur, Numéro 6, p.28-56, 1991.

[LAT 92] LATOUR B., Aramis ou l'amour des techniques, La découverte, Paris, 248 pages, 1992.

[LAW 04] LAW John., URRY J., “Enacting the Social”, Economy and Society, vol.33, n³, p. 390-410, 2004.

[MAI 08] MAILLET T., Génération participation: de la société de consommation à la société de la participation, MM2, Paris, 252 pages, 2008.

[MUS 07] MUSSO P., PONTOU L., SEULLIET E., Fabriquer le Futur, Pearsons Education France, Le Village Mondial, Paris, 307 pages, 2007.

[ROG 95] ROGERS E., Diffusion of innovations, The Free Press, New-York, 518 pages, 1995.

[SFE 02] SFEZ L., Technique et idéologie, Seuil, Paris, 323 pages, 2002.

[SIM 17] SIMON L, COHENDET P, SARAZIN B, Les communautés d'innovation: De la liberté créatrice à l'innovation organisée, EMS, Paris, 277 pages, 2017.

[SOU 17] SOULIER E, AUDRAN J., Communautés de pratiques et management de la formation, UTBM, Collection environnement et technologie, 328 pages, 2017.

[VON 86] VON HIPPEL E., "Lead Users: A Source of Novel Product Concepts, Management Science”, Vol 32, $n^{\circ} 7, p$ 791-806, 1986.

[WEN 98] WENGER E., Communities of Practice: Learning, Meaning, and Identity. Cambridge, University Press, Cambridge, 318 pages, 1998. 